\title{
Fever, bacterial zoonoses, and One Health in sub-Saharan Africa
}

\author{
Authors: Manuela Carugati, ${ }^{A}$ Kajiru G Kilonzo ${ }^{B}$ and John A Crump ${ }^{C}$
}

\begin{abstract}
Although often underappreciated, a number of bacterial zoonoses are endemic in Africa. Of these, brucellosis, leptospirosis, Q fever, and rickettsioses are responsible for a substantial proportion of febrile illness among patients seeking hospital care. In this paper, we discuss the aetiology, epidemiology, clinical presentation, diagnosis, treatment and prevention of these bacterial zoonoses. To prevent and control bacterial zoonoses, strategies targeting both animals and humans are crucial. These may lead to better outcomes than strategies based exclusively on treatment of human infections. Such strategies are referred to as the 'One Health' approach; the collaborative effort of multiple disciplines to attain optimal health for people, animals and the environment.
\end{abstract}

KEYWORDS: Sub-Saharan Africa, zoonoses, fever, One Health, brucellosis

\section{The patient with fever in sub-Saharan Africa}

Fever is a common symptom among patients seeking healthcare in sub-Saharan Africa where it may occur with or without localising features. ${ }^{1}$ With declines in malaria incidence worldwide and the wider availability of malaria diagnostic tests, the problem of malaria over-diagnosis has become apparent. ${ }^{2,3}$ Studies to describe major non-malaria causes of severe febrile illness in sub-Saharan Africa have identified the importance of bacterial bloodstream infections, HIV coinfections such as cryptococcal disease and disseminated tuberculosis, arbovirus infections and bacterial zoonoses such as brucellosis, leptospirosis, Q fever and rickettsioses. ${ }^{4,5}$ For example, research among 870 inpatients admitted with fever in northern Tanzania showed that malaria was the cause of fever in $14(1.6 \%)$ participants, while bacterial,

Authors: Aadjunct assistant professor, Duke University, Durham, USA and consultant, Fondazione IRCCS Ca' Granda Ospedale Maggiore Policlinico, Milan, Italy; ${ }^{B}$ head of department of internal medicine and senior lecturer, Kilimanjaro Christian Medical Centre, Moshi, United Republic of Tanzania; ' McKinlay professor of global health and co-director Otago Global Health Institute, University of Otago, Dunedin, New Zealand and adjunct professor of medicine, pathology and global health, Duke University, Durham, USA mycobacterial and fungal bloodstream infections accounted for $85(9.8 \%), 14(1.6 \%)$ and $25(2.9 \%)$ infections, respectively. Arboviral infections were diagnosed in 55 (7.9\%) patients enrolled in the study. Bacterial zoonoses were identified among 118 (26.2\%) patients; 16 (13.6\%) had brucellosis, 40 (33.9\%) had leptospirosis, $24(20.3 \%)$ had Q fever, 36 (30.5\%) had spotted fever group rickettsioses and 2 (1.8\%) had typhus group rickettsioses (Fig 1). ${ }^{6}$

\section{Brucellosis}

\section{Aetiology}

Brucellosis is caused by Brucella species, Gram-negative coccobacilli. Of a larger number of Brucella species with reservoirs in livestock and other animals, $B$ abortus, $B$ canis, $B$ melitensis and $B$ suis are recognized to cause human disease. The majority of human brucellosis worldwide is caused by $B$ melitensis. ${ }^{7}$

\section{Reservoir, source and mode of transmission}

Goats and sheep are the major reservoirs of $B$ melitensis. Cattle are the major reservoir of $B$ abortus, while $B$ canis is mainly found in canines, and $B$ suis in domestic and feral swine. In areas where cattle coexist with goats or are fed sheep offal, cattle can be infected with $B$ melitensis. Brucella localises to the reproductive tract and mammary glands of animals and may be present in the blood, reproductive tract secretions, milk and meat. Humans acquire the infection through direct contact, airborne transmission during aerosolisation and foodborne transmission. Risk factors for human brucellosis in sub-Saharan Africa include assisting goat or sheep births and having contact with cattle. Consuming boiled or pasteurised dairy products has been found to be protective against brucellosis. ${ }^{8}$

\section{Incidence, complications and death}

While it is well established that brucellosis is endemic in north Africa, the incidence of brucellosis is uncertain in many subSaharan African countries. ${ }^{4,9}$ A study from northern Tanzania demonstrated that brucellosis incidence was stable at 35 cases per 100,000 person-years during 2007-2008 and 33 cases per 100,000 person-years during $2012-2014 .^{10}$ Prevelance estimates for chronic brucellosis are lacking. Data from retrospective studies indicate that the case fatality ratio for brucellosis is low, at approximately $1 \%{ }^{7}$ During pregnancy, brucellosis can cause spontaneous abortion and fetal infection. 


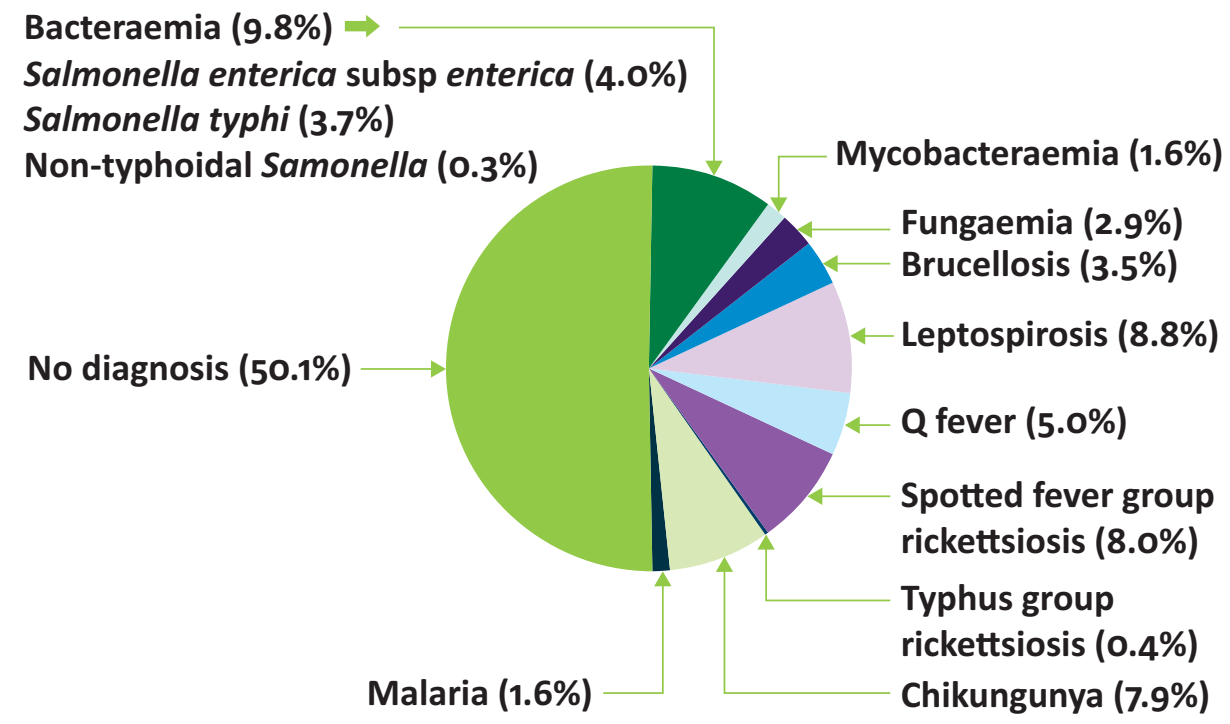

Fig 1. Causes of fever in inpatients, Moshi, Tanzania, 2007-2008.

Adapted with permission from Crump JA, Morrissey AB, Nicholson WL et al. Etiology of severe non-malaria febrile illness in northern Tanzania: a prospective cohort study. PloS Negl Trop Dis 2013;7:e2324.

\section{Clinical presentation}

The clinical presentation of brucellosis is protean. Acute infection often presents as a nonspecific febrile illness. Chronic forms of brucellosis include focal involvement of the osteoarticular, reproductive and central nervous system as well as hepatitis, liver abscesses, endocarditis, lobar pneumonia and pleural effusions. ${ }^{7}$

\section{Diagnosis}

Diagnostic confirmation of acute brucellosis relies upon isolation of Brucella species from culture of clinical specimens or a four-fold rise in Brucella antibody titre between acute and convalescent sera obtained at least 14 days apart. ${ }^{11}$ While blood culture sensitivity is $50-90 \%$, the sensitivity of bone marrow is $15-20 \%$ higher. The sensitivity and specificity for acute disease of Brucella paired serum agglutination tests exceed $90 \%$ in endemic areas. ${ }^{12}$ Neither the US Centers for Disease Control and Prevention nor the World Health Organization (WHO) has a specific case definition for chronic brucellosis. Finally, nucleic acid amplification tests are promising, but diagnostic performance varies substantially with the type of samples tested and the extraction methods used. ${ }^{13}$ Using blood cultures and serology as reference standards, the sensitivity of Brucella spp nucleic acid amplification tests (NAATs) has been reported as $61.0 \%$ in whole blood samples, $94.0 \%$ in serum samples and $97.1 \%$ in tissue samples from patients with a clinically active disease. . $^{14,15}$

\section{Treatment}

Tetracyclines and a parenteral aminoglycoside, or tetracyclines and rifampin are recommended by WHO for treatment of human brucellosis. The treatment duration is 6 weeks, except for spondylitis and osteomyelitis where $8-12$ weeks of therapy is needed.

\section{Prevention and control}

Brucellosis prevention and control is based on interventions among both animals and humans. Animal interventions include vaccination of animal populations; testing animals for infection and slaughtering those infected; and pasteurisation of dairy products. Interventions among humans include use of personal protective equipment, such as an overall apron, rubber gloves, boots, eye protection and mask for meat workers and for those dealing with aborting animals or animals undergoing parturition; and hygiene measures, minimally including rinsing of hands in approved disinfectants and washing with soap and water after contact with animals. Superficial injuries should be treated with an approved antiseptic, such as $1 \%$ chloramine solution. Intervention among humans are focused on specific groups such as abattoir workers and those dealing with aborting or parturient animals.

\section{Leptosirosis}

\section{Aetiology}

Leptospirosis is caused by spirochete bacteria in the genus Leptospira. There are 10 pathogenic species, and more than 250 pathogenic serovars. A large number of different Leptospira serogroups can cause human disease although predominant serogroups vary by year and by study.

\section{Reservoir, source and mode of transmission}

Pathogenic Leptospira have reservoirs in a wide range of animals including rodents and livestock and are shed in urine. ${ }^{16}$ The bacteria can survive for weeks to months in urine-contaminated water and soil. People are infected through direct or indirect contact with the urine of an infected animal. Rice farming, cleaning cattle waste, feeding cattle, farm work and increased exposure to cattle urine have been identified as risk factors for human leptospirosis in sub-Saharan Africa. ${ }^{17}$

\section{Incidence, complications and death}

A recent systematic review reported a prevalence of acute human leptospirosis in $2.3-19.8 \%$ of hospitalised patients with fever in Africa. ${ }^{18}$ Leptospirosis annual incidence in northern Tanzania has 
fluctuated from 75-102 per 100,000 people in 2007-2008 to 11-18 per 100,000 people in 2012-2014, suggesting dynamic transmission patterns. ${ }^{19}$ The case fatality ratio for leptospirosis is $5-15 \%$ among patients with severe illness. Among patients with pulmonary haemorrhage syndrome, the case fatality ratio can exceed $50 \%$. Leptospirosis during pregnancy can cause fetal death.

\section{Clinical presentation}

Most infections are asymptomatic. Of symptomatic infections, $90 \%$ present as a nonspecific acute febrile illness 5-14 days after exposure, while $10 \%$ progress to severe illness with multi-organ dysfunction including renal failure, liver failure and pulmonary haemorrhagic syndrome.

\section{Diagnosis}

The diagnosis of leptospirosis is based on either a four-fold rise in Leptospira antibody titre between acute and convalescent sera obtained at least 14 days apart, or a single reciprocal titre of $\geq 1: 800$ measured by agglutination test. ${ }^{20}$ The isolation of Leptospira species from culture of clinical specimens is also a valid but insensitive diagnostic method. Due to the transience of leptospires in body fluids, whole blood should be cultured during the first week, and urine after the first week of illness. Finally, NAAT allows early detection of Leptospira with reasonable sensitivity and specificity. ${ }^{21}$

\section{Treatment}

Recommended treatments for leptospirosis are doxycycline or amoxicillin in case of mild disease, and penicillin G or ceftriaxone in severe disease. In patients with a high clinical suspicion, antimicrobial treatment should be initiated as soon as possible without waiting for laboratory confirmation. The duration of treatment in severe disease is usually 7 days.

\section{Prevention and control}

Leptospirosis prevention and control is based on interventions among both animals and humans. Intervention among animals include animal vaccination and rodent control. Preventing leptospirosis in humans is based on avoiding swimming or wading in water that might be contaminated with animal urine; use of personal protective equipment, such as an overall apron, rubber gloves, boots and eye protection for people at occupational risk, such as abattoir workers and dairy farmers; and personal hygiene. Personal hygiene measures include hand washing after using the toilet or handling animals, covering skin breaks with waterproof coverings, avoiding smoking, drinking or eating when handling livestock, and washing clothes after handling stocks.

\section{Q fever}

\section{Aetiology}

Q fever is caused by the obligate intracellular bacterium Coxiella burnetii, which can survive in harsh environments in a spore-like form.

\section{Reservoir, source and mode of transmission}

Goats, sheep and cattle are the main reservoirs of $C$ burnetii. Infected animal birth products, urine, faeces and milk contain C burnetii, and spore-like forms contaminate dust. As a consequence, people can be infected by direct contact with milk, urine, faeces, and afterbirth of infected animals; breathing dust contaminated by infected animals; and consuming unpasteurised dairy products of infected animals. Transmission through blood transfusion, from a pregnant woman to her fetus, and sexual transmission have been reported.

\section{Incidence, complications and death}

C burnetii seroprevalence is $11-33 \%$ among small ruminants, $\leq 13 \%$ among cattle, and $<8 \%$ among humans in African studies. ${ }^{22,23}$ In a human cohort study, Q fever accounted for $5 \%$ of febrile illness hospitalisations. ${ }^{24} \mathrm{Q}$ fever is rarely fatal. An exception is Q fever related myocarditis that has a $25 \%$ case fatality ratio. The frequency of pregnancy complications in women developing $\mathrm{Q}$ fever is uncertain, although fetal death has been documented.

\section{Clinical presentation}

Of people infected with C burnetii, $50 \%$ will develop symptoms. Acute infection is characterised by fever usually accompanied by rigors and myalgia 2-3 weeks after exposure. Acute infection can be complicated by hepatitis, pneumonia and meningoencephalitis. Less than $5 \%$ of people infected with $C$ burnetii develop a chronic infection that could persist for more than 6 months. The most common presentations of chronic Q fever are endocarditis, particularly in persons with underlying valvular disease, and vascular manifestations, including infection of vascular protheses and aortic aneurysm. Less frequent presentations of chronic Q fever include osteomyelitis and hepatitis.

\section{Diagnosis}

In the presence of clinical symptoms, the diagnosis of acute and chronic $\mathrm{Q}$ fever may include the detection of $C$ burnetii by culture, polymerase chain reaction or immunohistochemistry. C burnetii culture requires biosafety level three laboratories and involves the inoculation of specimens onto conventional cell cultures, embryonated hen yolk sacs or laboratory animals. C burnetii NAAT can be applied to a wide range of clinical samples; its sensitivity is higher when applied to whole blood samples within 2 weeks of symptom onset. C burnetii immunohistochemistry in tissue can be performed using several techniques including immunoperoxidase staining, a capture enzyme-linked immunosorbent assay, an enzyme-linked immunosorbent fluorescence assay system, or monoclonal antibodies. Finally, serologic testing based on indirect immunofluorescent assay (IFA) can support the diagnosis of acute and chronic $\mathrm{Q}$ fever. The demonstration of a four-fold rise in phase II immunoglobulin $\mathrm{G}$ (IgG) by IFA between acute and convalescent serum samples taken 4 weeks apart is used to confirmed the diagnosis of acute $\mathrm{Q}$ fever. In contrast to acute infection, chronic $Q$ fever is characterised by phase I IgG titres typically equal or greater than 1:1,024. ${ }^{25}$ 


\section{Treatment}

Patients with acute $\mathrm{Q}$ fever should receive doxycycline for 14 days. Patients with chronic $Q$ fever should be treated with doxycycline and hydroxychloroquine for 18-24 months.

\section{Prevention and control}

Prevention and control of $\mathrm{Q}$ fever can include animal, environmental, and human interventions. ${ }^{26}$ Animal interventions include animal vaccination to reduce the shedding of the organisms and pasteurisation of dairy products. Environmental interventions are focused on reducing the risk of transmission of $C$ burnetii to human communities and include placement of high vegetation barriers around animal holdings; indoor parturition, safe disposal of parturition materials and safe disposal of hides and offal; and reduction of the aerosolisation by increasing soil moisture through irrigation or concreting surfaces. Human interventions are focused on the minimisation of the human contact with infected materials. Contaminated materials should be handled in ways that minimise the risk of aerosolisation. Personal protective equipment, such as an overall apron, rubber gloves, boots, eye protection and respirator mask rated $\mathrm{N} 95$ or higher should be worn in case of potential exposure to infected materials.

\section{Rickettsiosis}

\section{Aetiology}

Rickettsial infections are caused by various bacterial species from the genus Rickettsia. Rickettsia spp are classically divided into spotted fever group (SFG) and typhus group. Several rickettsial pathogens have been reported in sub-Saharan Africa.

\section{SFG rickettsioses}

Among the SFG rickettsioses, $R$ aeschlimanii, $R$ africae (African tick-bite fever), $R$ conorii (Mediterranean spotted fever), $R$ sibirica (lymphangitis-associated rickettsiosis), and $R$ felis are the most often reported from Africa. ${ }^{27}$

\section{Reservoir, source and mode of transmission}

SFG rickettsioses reservoirs include dogs and ruminants. While $R$ aeschlimanii, $R$ africae, $R$ conorii and $R$ sibirica are tick-borne infections, and $R$ felis is flea-borne. Organisms can be transmitted by bites from these vector species or by inoculating infectious fluids or faeces from vectors into the skin. Inhaling or inoculating conjunctiva with infectious material may also cause infection for some of these organisms.

\section{Incidence, complications and death}

Spotted fever group rickettsioses accounted for $8.0 \%$ of the causes of fever in a population of 870 febrile inpatients in Tanzania. ${ }^{24}$ Case fatality ratio ranges from $1.0-4.0 \%$ for typhus group rickettsiosis. ${ }^{28,29}$

\section{Clinical presentation}

Symptoms develop 6-10 days after the bite and may include fever, headache, muscle pain, macular or maculopapular rash, local lymphadenopathy and an eschar at the bite site. Mediterranean spotted fever may present with a single eschar, whereas multiple eschars may be present in African tick-bite fever.

\section{R typhi}

$R$ typhi (murine typhus or endemic typhus) is part of the typhus group rickettsioses and has been reported in sub-Saharan Africa.

\section{Reservoir, source and mode of transmission}

Rodents are the reservoir of $R$ typhi. $R$ typhi is transmitted to humans by fleas. Specifically, humans become infected when flea faeces containing $R$ typhi contaminate disrupted skin or are inhaled into the respiratory tract.

\section{Incidence, complications and death}

Typhus group rickettsioses accounted for $0.5 \%$ of the causes of fever in a population of 870 febrile inpatients in Tanzania. ${ }^{24}$ Endemic typhus is often unrecognised and misdiagnosed and is rarely associated to adverse outcomes.

\section{Clinical presentation}

Endemic typhus is typically a mild disease characterised by fever, headache and a transient rash.

\section{Diagnosis}

The diagnosis of rickettsiosis is based on the detection of Rickettsia spp by cell culture of blood and tissue specimens, immunohistochemical methods applied to tissue specimens, or blood and tissue specimen NAAT. While NAAT sensitivity in blood is low, NAAT of biopsy of eschar or rash material is sensitive. ${ }^{30}$ Furthermore, a four-fold rise in Rickettsia spp antibody titre between acute and convalescent sera obtained at least 14 days apart measured by an IFA is diagnostic. ${ }^{31}$ However, because of cross-reactivity of immune responses to rickettsial antigens, antibodies are group-specific but not species-specific.

\section{Treatment}

Doxycycline is the treatment of choice for rickettsioses. Treatment of patients with possible rickettsioses should be started when disease is suspected and should not await confirmatory testing, as certain infections can be rapidly progressive. Treatment duration is 7 days.

\section{Prevention and control}

Rickettsioses prevention is based on both vector and human interventions. Vector interventions consist in the use of insecticides. Human interventions are based on limiting the exposure to the vectors. This goal could be achieved wearing protective clothing and using insect repellent, such as diethyl-m-toluamide and permethrin, when undertaking activities where human contact with ticks, mites or fleas may occur such as during bushwalking and camping in infected areas. 
Table 1. Tests for the diagnosis of brucellosis, leptospirosis, $Q$ fever and rickettsioses

$\begin{array}{lllll} & \text { Culture } & \begin{array}{l}\text { Nucleic acid } \\ \text { amplification }\end{array} & \text { Serology } \\ \text { Brucellosis } & X & X & \text { Comments } \\ \text { Culture: bone marrow cultures are more sensitive than blood cultures. } \\ \text { NAAT: sensitivity is highest in tissue samples. } \\ \text { Leptospirosis } X\end{array}$

IgG = immunoglobulin G; NAAT = nucleic acid amplification test.

\section{Bacterial zoonoses and One Health approaches}

Although not widely appreciated or studied, bacterial zoonoses are likely to be endemic in many African countries and cause substantial human morbidity and mortality. ${ }^{6,32}$ In addition to causing human infections, bacterial zoonoses have important effects on the livelihoods of rural communities through their impacts on livestock fertility, growth, productivity and survival. ${ }^{7}$ Here, close contact with animals and their environments heightens risk for infection, human and livestock healthcare services are limited, and livestock represent a major source of nutrition and income for families. ${ }^{33}$ Despite their substantial impact on both human and animal health, bacterial zoonoses have been neglected because the burden of disease is poorly quantified is borne largely by disadvantaged populations with little political voice in low-resource areas. One Health is the collaborative effort of multiple disciplines to attain optimal and equitable health for people, animals and the environment. ${ }^{34}$ Bacterial zoonoses lend themselves to One Health approaches to management, prevention and control.

For clinicians working in low-resource settings, bacterial zoonoses present a number of challenges. From a diagnostic perspective difficulties include limited healthcare provider awareness; ${ }^{35}$ non-specific clinical presentation and frequent misdiagnosis with malaria; ${ }^{36}$ prior antimicrobial administration that may hamper the ability to detect bacteria; ${ }^{37}$ presentation later in the course of the illness when the bacteraemic phase has passed; ${ }^{37}$ lack of accurate tests at point-of-care and unavailability of confirmatory tests; ${ }^{38}$ and the limited diagnostic accuracy of single sample serology. ${ }^{39}$ Regarding patient management challenges, WHO Integrated Management of Adolescent and Adult Illness (IMAI) District Clinician Manual algorithms for severe febrile illness recommend ceftriaxone or ampicillin plus gentamicin. ${ }^{40}$ However, while these regimens would be effective for leptospirosis, they are not suitable for the treatment of brucellosis, Q fever and rickettsiosis.

Livestock and environmental interventions have the potential to effectively reduce the magnitude of bacterial zoonotic infections from the animal and environmental reservoirs, generating benefits to all who are epidemiologically linked with the source of the infection. ${ }^{30}$ The prevention and control of brucellosis, leptospirosis, Q fever and rickettsiosis is centred on approaches targeting animal reservoirs, environmental sources, vectors and humans. When implemented, such approaches contribute to better outcomes for human and animal health than those based exclusively on treatment of human infections. ${ }^{30}$ Both clinicians' awareness and resources for the development and implementation of control measures will be crucial for addressing bacterial zoonoses in Africa.

\section{Acknowledgements}

We acknowledge Prof Matthew P Rubach, Robert Chuwa, Dr Kate M Thomas and the staff of Duke Collaboration at Kilimanjaro Christian Medical Centre (Moshi, United Republic of Tanzania).

\section{Table 2. Treatment recommendations for brucellosis, leptospirosis, Q fever and rickettsioses.}

\section{Treatment}

Brucellosis

Leptospirosis

Mild disease: doxycycline or amoxicillin.

Severe disease: penicillin $\mathrm{G}$ or ceftriaxone.

Q fever $\quad$ Acute $Q$ fever: doxycycline.

Chronic Q fever: doxycycline and hydroxychloroquine.

Rickettsiosis Doxycycline.

\section{Duration}

6 weeks.

If spondylitis and osteomyelitis, 8-12 weeks.

7 days.

Acute $Q$ fever: 14 days.

Chronic Q fever: 18-24 months.

7 days. 


\section{References}

1 Feikin DR, Olack B, Bigogo GM et al. The burden of common infectious disease syndromes at the clinic and household level from population-based surveillance in rural and urban Kenya. PLoS One 2011;6:e16085.

2 Murray C], Ortblad KF, Guinovart C et al. Global, regional, and national incidence and mortality for HIV, tuberculosis, and malaria during 1990-2013: a systematic analysis for the global burden of disease study 2013. Lancet 2014;384:1005-70.

3 Reyburn H, Mbatia R, Drakeley C et al. Overdiagnosis of malaria in patients with severe febrile illness in Tanzania: a prospective study. BMJ 2004;329:1212-5.

4 Reddy EA, Shaw AV, Crump JA. Community-acquired bloodstream infections in Africa: a systematic review and meta-analysis. Lancet Infect Dis 2010;10:417-32.

5 Maze MJ, Bassat Q, Feasey NA et al. The epidemiology of febrile illness in sub-Saharan Africa: implications for diagnosis and management. Clin Microbiol Infect 2018;24:808-14.

6 Crump JA, Ramadhani HO, Morrissey AB et al. Invasive bacterial and fungal infections among hospitalized HIV-infected and HIV-uninfected adults and adolescents in northern Tanzania. Clin Infect Dis 2011;52:341-8.

7 Corbel MJ. Brucellosis in humans and animals. Geneva: World Health Organization, 2006. www.who.int/csr/resources/ publications/Brucellosis.pdf.

8 Cash-Goldwasser S, Maze M], Rubach MP et al. Risk factors for human brucellosis in northern Tanzania. Am J Trop Med Hyg 2018;98:598-606.

9 Dean AS, Crump L, Greter $\mathrm{H}$ et al. Global burden of human brucellosis: a systematic review of disease frequency. PLoS Negl Trop Dis 2012;6:e1865.

10 Carugati M, Biggs HM, Maze M] et al. Incidence of human brucellosis in the Kilimanjaro region of Tanzania in the periods 2007-2008 and 2012-2014. Trans R Soc Trop Med Hg 2018;112:136-43.

11 Centers for Disease Control and Prevention. Brucellosis 2010 case definition. Atlanta: CDC. wwwn.cdc.gov/nndss/conditions/ brucellosis/case-definition/2010/.

12 Rubach MP, Halliday JEB, Cleaveland S. Brucellosis in low-income and middle-income countries. Curr Opin Infect Dis 2013;26:404-12.

13 Araj GF. Updates on laboratory diagnosis of human brucellosis. Int J Antimicrob Agents 2010;36S:S12-7.

14 Morata P, Queipo-Ortuno MI, Reguera JM et al. Diagnostic yield of a PCR assay in focal complications of brucellosis. J Clin Microbiol 2001;39:3743-6.

15 Zerva L, Bourantas K, Mitka S et al. Serum is the preferred clinical specimen for diagnosis of human brucellosis by PCR. J Clin Microbiol 2001:39:1661-4.

16 Allan KJ, Halliday JEB, Moseley M et al. Assessment of animal hosts of pathogenic Leptospira in northern Tanzania. PLoS Negl Trop Dis 2018:12:e0006444.

17 Maze MJ, Cash-Goldwasser S, Rubach MP et al. Risk factors for human acute leptospirosis in northern Tanzania. PLoS Negl Trop Dis 2018;12:e0006372.

18 Allan KJ, Biggs HM, Halliday JEB et al. Epidemiology of leptospirosis in Africa: a systematic review of a neglected zoonosis and a paradigm for 'One Health' in Africa. PLoS Negl Trop Dis 2015;9:e0003899.

19 Maze MJ, Biggs HM, Rubach MP et al. Comparison of the estimated incidence of acute leptospirosis in the Kilimanjaro region of Tanzania between 2007-08 and 2012-14. PLoS Negl Trop Dis 2016;10:e0005165.

20 Centers for Disease Control and Prevention. Leptospirosis 2013 case definition. Atlanta: CDC. wwwn.cdc.gov/nndss/conditions/ leptospirosis/case-definition/2013/.
21 Mussoa D, Bernard LS, Musso D et al. Laboratory diagnosis of leptospirosis: a challenge. J Microbiol Immunol Infect 2013:46:245-52.

22 Vanderburg S, Rubach MP, Halliday JEB et al. Epidemiology of Coxiella burnetii infection in Africa: a OneHealth systematic review. PloS Negl Trop Dis 2014;8:e2787.

23 Wardrop NA, Thomas LF, Cook EA et al. The sero-epidemiology of Coxiella burnetii in humans and cattle, Western Kenya: evidence from a cross-sectional study. PLoS Negl Trop Dis 2016;7:e0005032.

24 Prabhu M, Nicholson WL, Roche AJ et al. Q fever, spotted fever group and typhus group rickettsioses among hospitalized febrile patients in northern Tanzania. Clin Infect Dis 2011;53:e8-15.

25 Anderson A, Bijlmer $\mathrm{H}$, Fournier PE et al. Diagnosis and management of Q fever, United States, 2013. MMWR 2013;62:1-23.

26 Clark NJ, Soares Magalhaes RJ. Airborne geographical dispersal of $Q$ fever from livestock holdings to human communities: a systematic review and critical appraisal of evidence. BMC Infect Dis 2018;18:218.

27 Parola P, Raoult D. Tropical rickettsioses. Clin Dermatol 2006:24:191-200.

28 Taylor AJ, Paris DH, Newton PN. A systematic review of mortality from untreated scrub typhus. PLoS Negl Trop Dis 2015;9:e0003971.

29 Adjemian J, Parks S, McElroy K et al. Murine typhus in Austin, Texas, USA, 2008. Emerg Infect Dis 2010:16:412-7.

30 Parola P, Paddock CD, Socolovschi C et al. Update on tick-borne rickettsioses around the world: a geographic approach. Clin Microbiol Rev 2013;26:657-702.

31 Centers for Disease Control and Prevention. Rocky Mountain Spotted Fever 2008 case definition. Atlanta: CDC. wwwn.cdc.gov/nndss/ conditions/rocky-mountain-spotted-fever/case-definition/2008/.

32 Grace D. Mapping of poverty and likely zoonoses hotspots. Zoonoses project 4. Report to the UK department for international development. Nairobi: International Livestock Research Institute. http://hdl.handle.net/10568/21161.

33 Cleaveland S, Sharp J, Abela-Ridder B et al. One Health contributions towards more effective and equitable approaches to health in low- and middle-income countries. Phil Trans $R$ Soc $B$ 2017;372:20160168.

34 Centers for Disease Control and Prevention. One Health basics. Atlanta: CDC. www.cdc.gov/onehealth/basics/index.html

35 Zhang HL, Mnzava KW, Mitchell ST et al. Mixed methods survey of zoonotic disease awareness and practice among animal and human healthcare providers in Moshi, Tanzania. PLoS Neglect Trop Dis 2016;10:e0004467.

36 Crump JA, Morrissey AB, Nicholson WL et al. Etiology of severe non-malaria febrile illness in northern Tanzania: a prospective cohort study. PloS Negl Trop Dis 2013;7:e2324.

37 Levett PN, Haake DA. Leptospira species. In: Mandell GL, Bennett JE, Dolin R (eds), Mandell, Douglas, and Bennett's principles and practice of infectious diseases, 7th ed. Churchill Livingstone, 2009.

38 Petti CA, Polage CR, Quinn TC et al. Laboratory medicine in Africa: a barrier to effective health care. Clin Infect Dis 2006;42:377-82.

39 De Glanville WA, Conde-Alvarez R, Moriyon I et al. Poor performance of the rapid test for human brucellosis in health facilities in Kenya. PLoS Negl Trop Dis 2017;11:e0005508.

40 World Health Organization. IMAI District Clinician Manual: Hospital care for adolescents and adults. Geneva: WHO, 2011. www.who.int/hiv/pub/imai/imai2011/en/.

Address for correspondence: Prof John A Crump, Centre for International Health, Dunedin School of Medicine, University of Otago, PO Box 56, Dunedin 9054, New Zealand.

Email: john.crump@otago.ac.nz 